\title{
Fault-Tolerant Quantum Computation with Local Gates
}

\author{
Daniel Gottesman* \\ T-6 Group, Los Alamos National Lab, Los Alamos, NM 87545 USA
}

\begin{abstract}
I discuss how to perform fault-tolerant quantum computation with concatenated codes using local gates in small numbers of dimensions. I show that a threshold result still exists in three, two, or one dimensions when next-to-nearest-neighbor gates are available, and present explicit constructions. In two or three dimensions, I also show how nearestneighbor gates can give a threshold result. In all cases, I simply demonstrate that a threshold exists, and do not attempt to optimize the error correction circuit or determine the exact value of the threshold. The additional overhead due to the fault-tolerance in both space and time is polylogarithmic in the error rate per logical gate.
\end{abstract}

\section{Introduction}

Quantum computation has the potential to offer vast speedups over classical computation. For instance, Shor's factoring algorithm [1] and Grover's database search algorithm [2] both offer great improvements over classical algorithms for the corresponding problems. However, quantum computers are likely to be highly susceptible to errors, whether caused by imperfect gates, decoherence due to interactions with the environment, or any other cause.

In classical computers, error correction is rarely necessary because the classical bits are stored using digital devices, which, at every time step, will restore the state of the system to a 0 or a 1 . They also are made up of a large number of smaller particles (electrons, usually), and therefore act as a simple (classical) repetition code.

The theory of quantum fault-tolerant computation [3, 4, 5, 6, 7, 8, 9, 10, 11] has developed in an attempt to allow a similar remedy to the buildup of errors in a quantum computer. Instead of performing our algorithm on some number of physical quantum bits (qubits), we implement it on a collection of logical qubits encoded using a somewhat larger number of physical qubits. The logical qubits live in a carefully chosen coding subspace of the full Hilbert space of the

*e-mail: gottesma@t6-serv.lanl.gov 
physical qubits. Then, by repeatedly performing an error correction algorithm during the computation, we can at every step restore (or nearly so) the data to the coding space, thus fending off errors.

In fact, by using concatenated quantum codes, we can produce an error threshold [4, 5, 6, 7, 10]: if the fundamental error rates per gate and per time step are below some threshold, we can perform arbitrarily long quantum computations with arbitrarily low logical error rate. This threshold is known to be at least $10^{-6}$ [5, 7], but is probably at least a few orders of magnitude higher [10]. Estimates range as high as 1/700 [11.

However, this result relies on a number of assumptions about the computer and errors, some of which are described below. One assumption is that gates can be performed between any pair of qubits. In practice, this may not be practical at all, since in a large computer, qubits will be constrained by the dimensionality of space to be far apart from each other. For instance, in Kane's proposal for a solid-state quantum computer using single-spin NMR [12], only adjacent qubits (in one or perhaps two dimensions) can directly communicate.

Luckily, this assumption is not critical to the result. As Aharonov and BenOr note [7, "the procedures ... can be made ...to operate only on nearest neighbors, by adding gates that swap between qubits." As we shall see, this is true, but we must be careful in how we design the computer. The ancilla qubits we need to perform error correction must be placed sufficiently near the computational qubits to be corrected, or too many errors will accumulate in the time necessary to move the interacting qubits together, taking us above the error threshold. Furthermore, when a number of levels of concatenation are used (as is necessary for long computations), some ancillas will necessarily be far away from the data, and we must be certain this does not destroy the threshold result.

\section{The Threshold Result}

First, I will review the usual threshold result. Each qubit is encoded with a concatenated quantum code, usually using the seven-qubit code 13. That is, each qubit is encoded as seven qubits via the mapping

$$
\begin{aligned}
|0\rangle \mapsto & |0000000\rangle+|1111000\rangle+|1100110\rangle+|1010101\rangle+ \\
& |0011110\rangle+|0101101\rangle+|0110011\rangle+|1001011\rangle \\
|1\rangle \mapsto \quad & |1111111\rangle+|0000111\rangle+|0011001\rangle+|0101010\rangle+ \\
& |1100001\rangle+|1010010\rangle+|1001100\rangle+|0110100\rangle,
\end{aligned}
$$

and each of those seven qubits is again encoded using the same map, and so on for $L$ levels.

The seven-qubit code has a number of properties that make it particularly favorable for fault-tolerant computation. The logical 0 of the seven-qubit code is the superposition of the even codewords of the (classical) Hamming code, whereas the logical 1 is the superposition of the odd codewords of the Hamming 


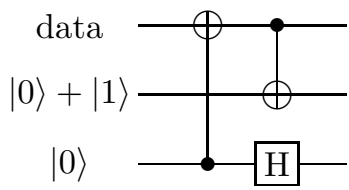

Figure 1: Error correction circuit for the seven qubit code. Each line represents a block of seven qubits, and each gate represents the same gate applied transversally on the block.

code. Therefore, to make a measurement of 0 vs. 1 , we need only measure each qubit in the block individually, and we will be able to determine the measurement result from the parity of the resulting Hamming codeword [9]. We do not need to perform an additional quantum error correction step before this measurement - phase errors will not affect the measurement result, and bit flip errors will show up as bit flip errors in the classical codeword, which can be corrected using classical methods. (The parity of the codeword should only be determined after this correction.)

In addition, it is easy to perform a number of fault-tolerant operations on the seven-qubit code. The Hadamard transform $H:|j\rangle \mapsto\left(|0\rangle+(-1)^{j}|1\rangle\right) / \sqrt{2}$, the phase gate $P:|j\rangle \mapsto i^{j}|j\rangle$, and the controlled NOT (or XOR) $|j, k\rangle \mapsto|j, j \oplus k\rangle$ can all be performed via simple transversal operations. A transversal operation only involves gates that interact the $r$ th qubit in a block with itself and the $r$ th qubits in other blocks. This prevents any errors from spreading within a block, so a single physical error cannot cause a whole block of seven to go bad.

The logical CNOT has another useful property: individual (or multiple) bit flip errors in the control block will propagate forwards, producing the corresponding bit flip errors in the target block, and phase errors in the target block will propagate backwards, producing the corresponding phase errors in the control block. In addition, the logical Hadamard transform will convert bit flip errors to phase errors and vice-versa, without changing the location of the errors.

We can take advantage of these facts to produce a simple fault-tolerant error correction circuit, shown in figure 11. We use the CNOT to copy the bit flip errors from the data block into an ancilla block, and measure the ancilla to see where those errors are. A similar procedure tells us the phase errors. The ancillas begin in the logical $|0\rangle$ and logical $|0\rangle+|1\rangle$ states so that when the data is correct, it is unaffected by the error correction procedure. From just one measurement, there is no way to tell whether an error is originally native to the data or to the ancilla, so we should repeat this procedure multiple times. Using some decision process, we then decide what the most likely error is and correct it.

Also, note that while we are measuring phase errors, bit flip errors can pass 
from the ancilla into the data, and vice-versa. If the errors are just singlequbit errors, this is not a major problem - we can correct them using the regular error-correction procedure. However, the process of creating the ancilla blocks may introduce correlated errors, and if those errors enter the data, it will be a serious problem. Therefore, we must also verify the ancilla blocks to eliminate such correlated errors. Precisely how we do this is not important for the discussion below, but it will certainly involve a number of additional ancilla qubits.

It is not difficult to see that all of the above properties hold equally well for the concatenated seven-qubit code. CNOTs, Hadamard, and phase gates can all be performed transversally on blocks of size $7^{L}$, and error correction can be performed by interacting a full block with additional ancilla blocks of size $7^{L}$. Since the concatenated seven qubit code is still a superposition of concatenated classical Hamming codewords, we can determine the error at all levels just from measuring these $7^{L}$-qubit blocks $[9$.

In order to complete a universal set of gates, we need an additional gate, such as the Toffoli gate [3] $(|i, j, k\rangle \mapsto|i, j, k \oplus i j\rangle)$. The construction of the Toffoli gate is somewhat involved, but requires three additional ancilla blocks encoded at the same level as the data, plus some "cat" states $(|00 \ldots 0\rangle+|11 \ldots 1\rangle)$ encoded with one less level. The construction also uses a number of Toffoli gates performed at the next lower level of concatenation. Therefore, construction of the Toffoli gate at level $L$ will first require the construction of Toffoli gates at level $L-1$, which requires the construction of Toffoli gates at level $L-2$, and so on.

Once we have all these tools, if we make a few assumptions about the form of the errors and our capabilities, we can prove the existence of an error threshold, below which arbitrarily long quantum computations are possible. At level $k$, there are a fixed number of places for errors to occur, and two errors are required to produce an error at level $k+1$ (our use of fault-tolerant procedures ensures this). Therefore, the error rate $P_{k+1}$ at level $k+1$ is related to the error rate $P_{k}$ at level $k$ by

$$
P_{k+1}=C P_{k}^{2},
$$

for some constant $C$, which essentially counts all possible combinations of level $k$ errors that are fatal at level $k$ (though most such combinations will be correctable at level $k+1$ ). Then

$$
P_{L}=(1 / C)\left(C P_{0}\right)^{2^{L}} .
$$

$P_{0}$, the error rate at level 0 , is the error rate on the physical qubits. If $P_{0}<$ $1 / C$, then $P_{L}$ will approach 0 extremely rapidly, as a double exponential in the number of levels. In fact, since the number of qubits required to encode at level $L$ is exponential in $L$, the error rate is a single exponential in the number of qubits (or equivalently, the number of qubits required is polylogarithmic in the desired error rate per gate). The value of the threshold is (at least) $1 / C$.

Because this scaling is so rapid, the result still holds true even if the number of possible places for errors increases, even exponentially, with the number of 
levels. Suppose

$$
P_{k+1}=C r^{k+1} P_{k}^{2}
$$

for constants $C$ and $r$. Then

$$
P_{L}=\frac{1}{C r^{2}}\left(C r^{2} P_{0}\right)^{2^{L}} / r^{L} .
$$

The threshold is still present, but is now $1 /\left(C r^{2}\right)$. This fact will be key to showing a threshold is still present when we use local gates. In this case, we cannot avoid the error rate per level increasing, but we will arrange the computer so that it only increases according to equation (5). Note that the final error rate is still a double exponential in the number of levels.

This does not completely prove the existence of a threshold for full-fledged fault-tolerant computation. It also must be possible to reliably create ancilla states for error correction at level $L$, and it must be possible to perform Toffoli gates at level $L$. Creating a reliable level $L$ ancilla requires first a number of reliable level $L-1$ ancillas, then a number of level $L-1$ gates. For instance, to create an encoded $|0\rangle$ state at level $L$, we take 7 level $L-1$ encoded $|0\rangle$ states and perform an appropriate circuit interacting them to produce the level $L$ state.

Since multiple blocks (of $7^{L-1}$ qubits) within the level $L$ block interact, this encoding network could introduce multiple correlated errors in the level $L$ block, so we must verify the encoded states. For instance, one way to do this is to compare them against each other (using essentially the regular error-correction procedure) - correlated errors in one block will be uncorrelated with errors in another block. This will produce a smaller number of more reliable ancillas, which we again compare against each other. With a constant number of rounds of comparison, we can reduce the chance of correlated errors to less than the chance of a similar number of errors arising individually (recall that uncorrelated errors are arising during the verification procedure as usual). Other methods will produce qualitatively similar results.

If the effective error rate in the level $L-1$ ancillas and the level $L-1$ gates is low enough, then we can create reliable level $L$ ancillas [10]. The procedure is self-similar - if creating a reliable ancilla at level $k$ (including all verification steps) requires a total of $N_{a}$ level $k-1$ ancillas 1 a level $k+1$ ancilla also requires $N_{a}$ level $k$ ancillas. Therefore, to create a level $L$ ancilla ultimately requires $N_{a}^{L}$ level 0 ancillas.

Essentially the same logic applies to the level $L$ Toffoli gate. The requirements for reliable preparation and reliable Toffoli gates will both somewhat lower the threshold, but will not destroy it.

Perhaps the most important assumption we make is that we can perform operations in parallel. Otherwise the situation becomes akin to spinning plates

\footnotetext{
${ }^{1}$ In the example verification procedure above, $N_{a}$ would need to include not just the seven level $k-1$ ancillas encoded for the original block, but also all of the ancillas used to create the blocks against which the original level $k$ ancilla is compared, and for the blocks against which they are compared, and so on.
} 
to keep them from falling - each requires a certain amount of time to spin, and once the number of plates becomes too large, we will not have time to spin all of them before the first one falls. The precise degree of parallelism we assume will heavily impact the error threshold. Generally I will assume maximum parallelism - all pairs of qubits that can interact can do so at the same time, provided no qubit participates in two different interactions at once - but since I will not be calculating an explicit error threshold, this assumption will not greatly impact the discussion.

Another important assumption is that errors are uncorrelated, both in time and in space. If there is a chance $p$ per time step that the whole computer will break down, we will not be able to perform more than about $1 / p$ computational steps on average. We can allow small-scale correlations without much damage (though the lower levels of the code will be less effective than expected in that case), but long-range correlations in the errors have the potential to cause serious problems. Systematic gate errors can also be tolerated, but may significantly lower the threshold [5].

We also require a supply of fresh qubits during the computation to act as ancillas during error correction. These ancillas provide a place for us to dump entropy - otherwise the Second Law of Thermodynamics would forbid arbitrarily long computations. In this paper, I shall assume that qubits can be initialized and erased in place. This appears to be a strict requirement - if a qubit has to move a long distance from where it is initialized to where it is used, it will likely be randomized by the time it arrives.

Note that all three of the above assumptions apply equally well to faulttolerant classical computation. The last could conceivably be circumvented by a careful use of irreversible gates, but the ability to perform an appropriate variety of gate is really just another form of the same assumption.

Some other assumptions are useful, but not necessary. For instance, we generally assume that errors may randomize the qubits, but will never cause them to leave the computational space. Since it is possible in principle to detect such a "leakage," we can remove this assumption by adding in "stop leak" gates that watch for such an error. It is also frequently convenient to assume we have the capability to make measurements on the qubits during the computation, and to rapidly perform (modest) classical computations between quantum steps. We can remove this assumption by simulating the classical computation with a quantum circuit (though it must follow the design principles of a classical faulttolerant computer). In the case of local quantum gates, we would intersperse quantum bits with regions designated for these classical computations (see, for instance, 14 for a one-dimensional classical fault-tolerant architecture). Having a reliable classical computer available considerably simplifies the task of building a fault-tolerant quantum computer, since we can assume our decision processes (such as for which error occurred) are reliable.

Another unnecessary assumption is that arbitrary pairs of qubits can communicate directly. In this paper, I shall show that it is sufficient to interact nearby pairs of qubits. Then by moving the data around, we can allow originally distant pairs of qubits to interact. We must, however, be careful that the 


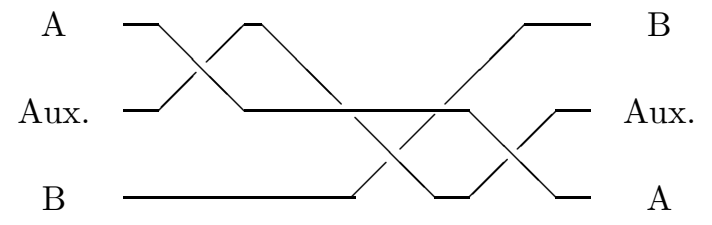

Figure 2: Network to fault-tolerantly swap two computational qubits

time required to do so is not too large, or by the time the qubits are brought together, they will both be erroneous.

\section{$3 \quad$ Swapping Qubits}

In order to perform effectively long-range interactions, we shall require the ability to move qubits around. We will accomplish this by swapping adjacent qubits. An appropriate series of swaps between adjacent qubits will allow us to perform an arbitrary permutation of the qubits. We will primarily be interested in cyclic rotations, moving a single qubit a distance $d$ (qubit 1 becomes qubit $d$, while qubit $s$ becomes qubit $s-1$ for $s=2, \ldots, d$ ), requiring $d-1$ swaps. To interact two qubits initially a distance $d+1$ apart, we perform one such rotation, bringing the first qubit adjacent to the second, then interact the two, then perform a second rotation, returning the first qubit to its original location. Altogether, we need $2(d-1)$ swaps for this interaction.

While we do not need to worry about the swap operation propagating preexisting errors (it swaps the errors along with the data), we do have to worry about errors in the swap gate itself, which could introduce correlated errors in the two qubits being swapped. To solve this problem, we introduce an auxiliary qubit between the two computational qubits A and B (which may themselves be ancillary to the primary computation). Then the following series of gates will swap A and B without ever interacting them directly:

$$
\begin{aligned}
& \text { 1. Swap }(1,2) \\
& \text { 2. Swap }(1,3) \\
& \text { 3. Swap }(2,3)
\end{aligned}
$$

(see figure 2). While the auxiliary qubit may acquire correlated errors with A or $\mathrm{B}$, that does not matter, since the value of the auxiliary qubit is completely immaterial.[ [ Note that this network requires next-to-nearest-neighbor gates.

\footnotetext{
${ }^{2}$ We might as well perform two unrelated quantum computations on this computer at the same time, with the auxiliary qubits for one being the computational qubits for the other.
} 
To allow swaps between arbitrary pairs of neighboring computational qubits, we need to alternate computational qubits with auxiliary qubits, as in figure 3 a. Note that in two or more dimensions, we can manage with simple nearestneighbor gates by arranging cul-de-sacs where we can temporarily store one computational qubit while moving another past it (figure 3b). For instance, to move a qubit A two positions up (figure 3 c), we simply slide the two (computational) qubits above it into the cul-de-sacs down and to the right from their normal positions, using regular nearest-neighbor swaps. Then move A up to its destination, and move the two displaced qubits into the computational slots down and to the left. With or without cul-de-sacs, moving a qubit a distance $d$ requires $O(d)$ gates.

The remainder of the protocol only requires swaps and other interactions between nearest neighbors. One might think the Toffoli gate would require next-to-nearest neighbor interactions, but in fact, it can be built up from oneand two-qubit gates [15]. Therefore, in two or more dimensions, we will be able to perform fault-tolerant computation with only nearest-neighbor interactions, whereas in one dimension, the inability to use cul-de-sacs to move the data out of the way requires us to go to next-to-nearest-neighbor interactions. However, in many almost one-dimensional systems (such as two parallel lines of qubits, or a single line with an occasional additional qubit on the side), we can again move to nearest-neighbor interactions.

In fact, since swap gates cannot propagate errors, it will likely be possible to use nearest-neighbor gates even in one-dimensional systems. However, since a single swap gate could introduce correlated errors on pairs of qubits in the same block, it might be necessary to use a concatenated code that corrects two errors per level instead of the concatenated seven-qubit code.

\section{Three Dimensions}

When our qubits lie on a three-dimensional cubic lattice, we use the arrangement of figure 1 for our computer. Each logical qubit has associated with it many ancillas. We arrange a single encoded data qubit on a plane with all of its ancillas. Then we stack the planes, aligning the data qubits in all of the planes. Therefore, to perform a tranversal interaction between adjacent data qubits, we do not need to move anything. To perform a transversal interaction between distant data qubits, we must first move them together. By treating each step in the move as a regular computational step, we can see that a computation involving $K$ logical qubits will be slowed down by a factor of at most $K$ computational steps. When $K$ is large, we will have to perform error correction at intermediate stages during the move, but this does not present any particular extra burden, since we have the ancillas for error correction constantly available.

In a single plane, we have many lines of qubits. One line will consist of the data block of $7^{L}$ qubits. Other lines will consist of ancillas of various forms and functions. After being used, an ancillas is reinitialized to be used again (a process which we are assuming can be done in place). The ancillas are aligned 
a)

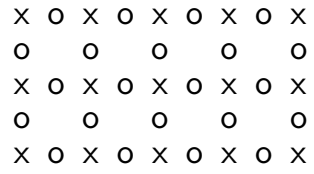

b)

$\mathrm{x} \times \mathrm{O} \times 0 \times 0 \times$

O C

$\times 0 \times 0 \times 0 \times 0 \times$

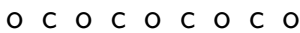

$\times 0 \times 0 \times 0 \times 0 \times$ c)

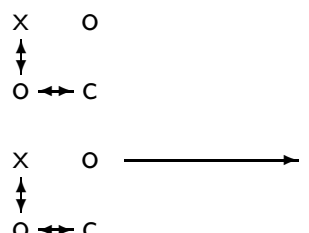

A 0

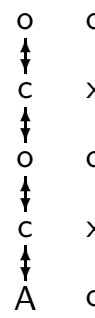

A 0

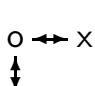

†

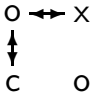

A $\mathrm{O}$

$c \leftrightarrow \mathrm{O}$

$\times \quad 0$

$C \leftrightarrow 0$

$\times \quad \mathrm{O}$

Figure 3: a) Computational qubits (x) arranged on a square lattice, interspersed by auxiliary qubits (o). b) adds cul-de-sacs (c) for moving qubits out of the way. c) Moving a data qubit (A) two positions.

a)

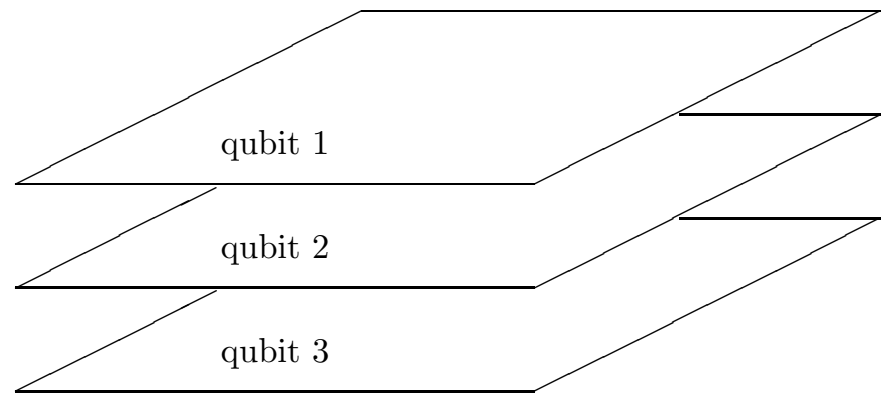

b)

\begin{tabular}{|c|c|c|c|c|c|c|c|c|c|c|c|c|c|c|c|c|c|c|c|c|}
\hline $\mathrm{d}$ & 0 & 0 & 0 & 1 & 0 & 0 & 0 & 1 & 0 & 0 & 0 & \begin{tabular}{|l|}
1 \\
\end{tabular} & 0 & 0 & 0 & 2 & 0 & 0 & 0 & 1 \\
\hline $\mathrm{d}$ & 0 & 0 & 0 & 1 & 0 & 0 & 0 & 1 & 0 & 0 & 0 & 1 & 0 & 0 & 0 & 2 & 0 & 0 & 0 & 1 \\
\hline $\mathrm{d}$ & 0 & 0 & 0 & 1 & 0 & 0 & 0 & 1 & 0 & 0 & 0 & 1 & 0 & 0 & 0 & 2 & 0 & 0 & 0 & \\
\hline $\mathrm{d}$ & 0 & 0 & 0 & 1 & 0 & 0 & 0 & 1 & 0 & 0 & 0 & 1 & 0 & 0 & 0 & 2 & 0 & 0 & 0 & 1 \\
\hline 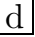 & 0 & 0 & 0 & 1 & 0 & 0 & 0 & 1 & 0 & 0 & 0 & 1 & 0 & 0 & 0 & 2 & 0 & 0 & 0 & 1 \\
\hline
\end{tabular}

Figure 4: a) The logical qubits of the computer lie on separate planes. b) Each plane has the data on one line, adjacent to ancillas at various levels. The letter $\mathrm{d}$ represents a data qubit, 0 is a qubit from a level 0 ancilla, 1 is from a level 1 ancilla, and so on. 
with the data so that all interactions take place along a single "interaction" axis (perpendicular to the arrangement of the data block), thus realizing the transversal nature of the interactions. The only time interactions will occur along the other axis (the "data" axis) is when an ancilla is being encoded (to create a "cat" state, for instance, or a level $k$ ancilla from level $k-1$ ancillas). Any such encoding will always be followed by verification steps.

The ancillas necessary for level 0 operations, including both error correction and the Toffoli gate, must be nearby the data - we cannot tolerate moving qubits a long distance for level 0 operations. Therefore we place the level 0 ancillas immediately adjacent to the data along the interaction axis. There are a fixed number $N_{o}$ of such ancillas, not increasing with the number of levels of encoding used in the computer (we only count the ancillas that directly interact with the data qubit). Depending on their function, these level 0 ancillas may have a number of different forms. They are frequently encoded in blocks of 7 , which interact with corresponding 7 -qubit blocks of the code. Level 0 ancillas which are adjacent along the data axis are completely independent — they interact with different blocks of the data, and no communication between them is necessary.

There are also a total of $N_{o}$ level 1 ancillas for level 1 operations and error correction on the data. We place the first of these after the last level 0 ancilla on the interaction axis.] Following the first level 1 ancilla, we place a number $N_{t}$ of level 0 ancillas, which are necessary for preparation and error-correction of the level 1 ancilla. After that comes another level 1 ancilla, followed by another set of level 0 ancillas, and so on. We require the ability to correct errors on the level 1 ancillas because they may have to move a considerable distance to interact with the data, and we may wish to preserve their state during the move.

After all of the level 1 ancillas, we place the first level 2 ancilla, followed by the level 0 ancillas necessary to maintain it. After those comes the first of the level 1 ancillas necessary to prepare and maintain the level 2 ancilla, then the level 0 ancillas to prepare and maintain the level 1 ancilla, then another level 1 ancilla, and so on. The level 2 ancilla requires a total of $N_{t}$ level 1 ancillas, and each has associated with it $N_{t}$ level 0 ancillas. Therefore, each level 2 ancilla requires at most $\left(N_{t}+1\right)^{2}$ lines of qubits for its support structure.

We follow this pattern as far as necessary - each level $k$ ancilla requires $N_{t}$ level $k-1$ ancillas, each of which requires $N_{t}$ level $k-2$ ancillas, each of which requires $N_{t}$ level $k-3$ ancillas, and so on. We can see that the total number of ancillas grows exponentially with level. This means that to interact with the data qubit, a level $k$ ancilla will have to move a distance $N^{k}$ for some constant $N$ (greater than $N_{t}$ and $N_{o}$ ). This means that the interaction takes $O\left(N^{k}\right)$ times as much time to occur as when we had long distance interactions, so the possibilities for error also increase exponentially with $k$. However, this merely recovers equation (5),

$$
P_{k+1}=C r^{k+1} P_{k}^{2},
$$

\footnotetext{
${ }^{3}$ Note that a "level $k$ ancilla" is used for operations or error correction at level $k$. When it is used for error correction, it may in fact be a state encoded at level $k+1$.
} 
which still has an error threshold. Therefore, we can perform fault-tolerant computation with local gates in three dimensions.

Note that a block encoded at level $k$ may have different error rates at level 0 , level 1 , and so on (a level 0 error is a single erroneous qubit; in a level 1 error, a whole block of 7 has gone bad). It is important that only the probability of level $k$ errors, and not the probability of level 0 errors (or errors at another fixed level), increases with $k$. If a level $k$ ancilla experiences an exponential (or even linear) increase in the probability of level 0 errors, equation (5) will not be valid. It is to solve this potential problem that we need to be able to correct errors on the ancillas as well as prepare them. For instance, we might move the level $k$ ancilla $s$ spaces towards the data, then perform error correction using the local extra ancillas, staving off low-level errors, then resume its movement. Note that to perform level $l$ error correction on the ancilla (with $l<k$ ), we may need to move level $l$ ancillas around to bring them next to the level $k$ ancilla. However, these level $l$ ancillas will only have to move a distance at most $N^{l}$, fl so equation (5) holds. We should also perform low-level error correction on the data at the same time as on the ancilla, since the data is accumulating errors while it waits for the level $k$ ancilla to arrive. Some other ancillas may also contain important information, and we should perform error correction on them too.

Since level $L$ ancillas now have to move a distance $N^{L}$ to interact with the data, this protocol produces a slowdown by a factor of $O\left(N^{L}\right)$ relative to the usual fault-tolerant protocol. Since the procedure requires more stops for lowlevel error correction, which in turn means more ancillas, there will be a similar increase in the number of qubits needed. However, since the error rate is a double exponential in $L$, this means the additional overhead is only polylogarithmic in the desired error rate.

\section{Two Dimensions}

In two dimensions, we adopt a somewhat similar arrangement. Now the individual data qubits and their ancillas form lines, which are again aligned so that transversal gates between adjacent data qubits are straightforward (see figure 5).

The arrangement of the individual lines can also be seen in figure 5 . Next to each data qubit, we place the corresponding qubits from the $N_{o}$ level 0 ancillas. We do this for a block of seven data qubits (since we are using the seven-qubit code), and then place the level 1 blocks for the $N_{o}$ level 1 ancillas required to perform error correction and Toffoli gates at level 1 with the data. These blocks themselves contain, interspersed with the qubits in the level 1 blocks, the $N_{t}$ level 0 ancillas to create and maintain the level 1 ancillas. We repeat this pattern (level 1 data blocks next to $N_{t}$ level 1 ancilla blocks) seven times, then position the level 2 ancilla blocks with their support structures between the level 2 data blocks.

\footnotetext{
${ }^{4}$ Or perhaps $2 N^{l}$, since partway through the move two level $k$ ancillas could be adjacent.
} 


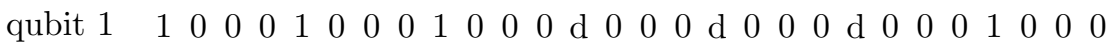

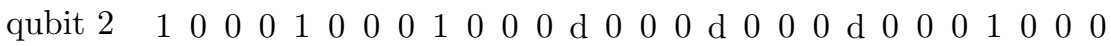

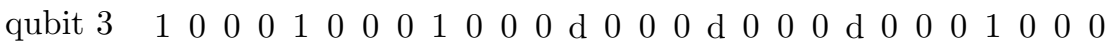

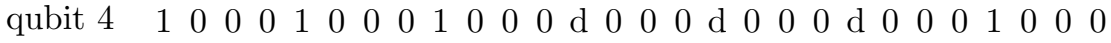

Figure 5: The logical qubits of the computer lie on separate lines. Within each line, ancillas are interspersed with the data qubits. Again, d represents a data qubit, 0 is from a level 0 ancilla, and 1 is from a level 1 ancilla.

As in the three dimensional case, this structure means that a level $k$ ancilla will have to move a distance $N^{k}$ to interact with the data (although $N$ may be bigger). Again, we may have to perform level $l$ error correction along the way (with $l<k$ ), but a level $l$ ancilla is never further than $N^{l}$ places away. We once again arrive with a recursion relation in the form (5), so we still have an error threshold.

\section{One Dimension}

Suppose we had just a two-qubit quantum computer. We could easily convert the two-dimensional model into a one-dimensional model by alternating qubits from the line associated with the first data qubit with the line associated with the second data qubit (so we would have a qubit from data block 1 followed by a qubit from data block 2 , then a level 0 ancilla qubit for data block 1 , then a level 0 ancilla qubit for data block 2, and so on). In this model, each ancilla will have to move exactly twice as far as in the two-dimensional case, so there will still be a threshold, though it will be half as large. To interact the two data qubits, we should perfectly align the blocks, so that qubit number 57 from block 1 is right next to qubit number 57 from block 2. However, if the logical qubits do not need to interact, there is no reason the blocks need to be aligned. We will still be able to perform error correction on the blocks separately, even if they are out of phase.

In the two-dimensional model, each data block with its ancillas (and the support structure for the ancillas) took up only a finite amount of space $\left(T^{L}\right.$ for some constant $T$ when there are $L$ levels altogether). That means that we can create an arbitrarily large one-dimensional quantum computer by placing these blocks of $T^{L}$ alongside each other.

However, to interact two adjacent blocks would require moving qubits a distance $T^{L}$ — too far to go without error correction. The solution is to move the support structure for the data block along with the data block itself, interleaving the two blocks as in the two-qubit example. We will probably have to stop during the move to do error correction, and the blocks will still be out of phase at this point. Since we do not need to interact the blocks to perform error correction, this is not a problem. We can bring the blocks into phase, interact them, then 
move them back apart.

All in all, this process will slow the computer down by an additional factor of $T^{L}$ (beyond the two-dimensional case). Again, this only results in an additional polylogarithmic slowdown. Therefore, even for the one-dimensional case, faulttolerant quantum computation is possible with local gates.

\section{Acknowledgements}

I would like to thank David DiVincenzo and particularly John Preskill for helpful conversations. This research is supported by the Department of Energy under contract W-7405-ENG-36.

\section{References}

[1] P. W. Shor, "Polynomial-time algorithms for prime factorization and discrete logarithms on a quantum computer," Proc. 35th Annual Symp. on the Foundations of Computer Science, p. 124 (IEEE Computer Society Press, Los Alamitos, CA, 1994), quant-ph/9508027.

[2] L. Grover, "Quantum mechanics helps in searching for a needle in a haystack," Phys. Rev. Lett. 79, 325 (1997).

[3] P. W. Shor, "Fault-tolerant quantum computation," Proc. 37th Ann. Symp. on the Foundations of Computer Science, p. 56 (IEEE Computer Society Press, Los Alamitos, CA, 1996), quant-ph/9605011.

[4] E. Knill and R. Laflamme, "Concatenated quantum codes," quant$\mathrm{ph} / 9608012$.

[5] E. Knill, R. Laflamme, and W. H. Zurek, "Threshold accuracy for quantum computation," quant-ph/9610011; E. Knill, R. Laflamme, and W. H. Zurek, "Resilient quantum computation," Science 279, 342 (1998); E. Knill, R. Laflamme, and W. H. Zurek, "Resilient quantum computation: error models and thresholds," Proc. Royal Soc. Lond. A 454, 365 (1998), quant$\mathrm{ph} / 9611025$.

[6] A. Y. Kitaev, "Quantum error correction with imperfect gates," Quantum Communcation, Computing, and Measurement (Proc. 3rd Int. Conf. of Quantum Communication and Measurement), p. 181 (Plenum Press, New York, 1997).

[7] D. Aharonov and M. Ben-Or, "Fault-tolerant quantum computation with constant error," Proc. 29th Ann. ACM Symp. on Theory of Computing, p. 176 (ACM, New York, 1998), quant-ph/9611025.

[8] D. Gottesman, "Theory of fault-tolerant quantum computation," Phys. Rev. A 57, 127 (1998), quant-ph/9702029. 
[9] J. Preskill, "Fault-tolerant quantum computation," ch. 8 in Introduction to Quantum Computation and Information, eds. Hoi-Kwong Lo, Sandu Popescu, and Tim Spiller, p. 213 (World Scientific, New Jersey, 1998), quant-ph/9712048.

[10] D. Gottesman and J. Preskill, unpublished.

[11] C. Zalka, "Threshold estimate for fault-tolerant quantum computing," quant-ph/9612028.

[12] B. E. Kane, "A silicon-based nuclear spin quantum computer," Nature $\mathbf{3 9 3 ,}$ 133 (1998).

[13] A. M. Steane, "Error correcting codes in quantum theory," Phys. Rev. Lett. 77, 793 (1996).

[14] P. Gács, "Reliable computation with cellular automata," J. Comp. Sys. Sci. 32, 15 (1986).

[15] A. Barenco, C. H. Bennett, R. Cleve, D. P. DiVincenzo, N. Margolus, P. Shor, T. Sleator, J. A. Smolin, and H. Weinfurter, "Elementary gates for quantum computation," Phys. Rev. A 52, 3457 (1995), quant-ph/9503016. 